\title{
THRESHOLD MODELS OF INTERPERSONAL EFFECTS IN CONSUMER DEMAND*
}

\author{
Mark GRANOVETTER \\ State University of New York at Stony Brook, NY 11794, USA \\ Roland SOONG \\ Arbitron Ratings Company, New York, NY, USA
}

Received November 1983, final version received August 1985

\begin{abstract}
Whether one buys may be determined in part by how many others have. When the correlation is positive we refer to 'bandwagon' effects, and when negative, 'reverse bandwagons'. We construct demand schedules in the presence of such effects and, with simple assumptions about supply, investigate the existence of and approach to equilibrium. Stable price-quantity equilibria exist, but for many plausible parameter values, equilibria are asymptotically unstable, and system trajectories consist of cycles that can move, with slight parameter changes, via successive bifurcations into what has been called 'chaotic' dynamics, essentially indistinguishable from random noise. These conditions occur despite assumptions of perfect information, profit maximizing firms and utility maximizing individuals.
\end{abstract}

\section{Introduction}

Despite such notable exceptions as Veblen (1899), Duesenberry (1949) and Leibenstein $(1950,1976)$, interpersonal effects on consumer demand are rarely explicitly analyzed. The present paper concerns the impact on markets of a common interpersonal effect: that whether one buys a good depends on the number of previous buyers. We construct demand schedules resulting from this effect and consider some peculiarities in how supply and demand are then matched, and the implication for attainment of equilibrium price/quantity combinations. Our underlying assumptions are similar to Leibenstein's (1976) but the model differs. His analysis is static, and assumes that (p. 56) 'buyers have accurate knowledge of market conditions (of the total quantities demanded at every price)'. We consider dynamics explicitly, and require

\footnotetext{
*This paper has been improved by the helpful comments of Richard Day, Eric Leifer, Thomas Muench, Thomas Schelling, Harrison White, Christopher Winship and an anonymous referee. A first draft was completed in sabbatical facilities provided by the Department of Sociology, Harvard University, and the work was supported in part by a John Simon Guggenheim Memorial Foundation Fellowship and by National Science Foundation Grant no. SPI 81-65055, both to the first author.
} 
buyers to know only the volume purchased in the previous time period. The analysis is based on a general argument previously put forward as 'threshold models of collective behavior' [Granovetter (1978), Granovetter and Soong (1983)].

The interpersonal effects analyzed here are of two kinds. In the first, 'bandwagon effects' [cf. Leibenstein (1976)], the correlation between one's purchases and those of others is positive. This may occur because the utility of a good is directly enhanced by the number of others consuming it. There are three distinct reasons for such enhancement. One has to do with statusseeking, when people (p. 51) 'purchase a commodity in order to get into the "swim of things", in order to conform with those they wish to be associated with or in order to be fashionable or stylish'. A second is that some goods may depend for their utility on joint consumption by others. Eating in an empty restaurant is depressing; restaurants with windows facing the street fill visible tables first, to convey tó passersby a convivial atmosphere. A third direct effect on utility results when services or accessories for one's purchase are more readily and cheaply available the more others have purchased - as is well understood by those seeking warranty service for their 'off-brand' appliance or software for their unknown brand of personal computer.

Bandwagon effects may also occur because the number of previous purchasers is taken as a signal of product quality. Philip Nelson's (1970) distinction between 'search' and 'experience' goods is relevant, the latter being goods whose characteristics can only be known after use; in practice, however, one often relies on others' experiences as a screening device. The number of diners in a restaurant may be taken not only as a sign of conviviality, but also as a commentary on the quality of food. When buying new or unfamiliar products - as with first-time parents facing the market for disposable diapers - the number of others using a brand may be a large influence on purchases. The value of such screening is enhanced when a market is complexly differentiated and sufficient experience with all alternatives to make optimal judgements would be time-consuming and expensive, as with personal computers.

The opposite of a bandwagon effect occurs if the correlation between one's consumption and that of others is negative. These 'reverse bandwagons' have causes closely paralleling those already cited. Status-seeking may require avoidance of overly popular products: Leibenstein (1976) refers to 'snob effects'. Products such as clothing, cosmetics and works of art, where high style and panache are essential, are especially implicated. Enjoyment may be directly reduced as the number of other consumers increases. Empty restaurants or parks may be uninviting, but so are those that are jam-packed. While services and accessories may be scarce when purchases of a product are too few, they may also be unavailable or delayed when they are too many. Signaling interpretations seem less likely here than for forward 
bandwagons, as they imply a judgement that the more purchasers of a product, the lower its quality; an example is the 'contrarian' position in financial markets.

The same commodity may be subject both to bandwagon effects and their reverse, over different ranges of consumption volume. Restaurants, for example, appeared in discussions of both, and there may be many products that people are unwilling to purchase until some minimum number of others has, but that also becomes less appealing once some maximum number is exceeded.

\section{Threshold models of consumer behavior}

Begin with forward bandwagons. Assume that in each time period, a consumer must decide whether or not to purchase one unit of a given commodity, so that the number of purchasers equals the number of units purchased. ${ }^{1}$ For a fixed product price assume that each person is influenced by what proportion of some relevant group of others have bought the product, but that different people are influenced in different degrees. Characterize a person who will buy only after he sees $50 \%$ of others do so as having a 'threshold' of $50 \%$, and assume that once one's threshold is surpassed, he will purchase and continue to do so in each subsequent time period. We can depict the distribution of such thresholds as a probability density, $f(x)$, which we assume continuous.

Suppose the proportion who have purchased the product in a given period is $r(t)$. Purchasers at $(t+1)$ are just those whose threshold is less than or equal to $r$, and their proportion can be found by integrating the density, $f(x)$, to yield a cumulative distribution function (c.d.f.), $F(x)$. It follows that

$$
r(t+1)=F(r(t))
$$

At equilibrium we have $r(t)=F(r(t))$, so that a graph of $F$ against $r$ will display all equilibria as intersections of the curve with the $45^{\circ}$ line, as for the three equilibria in fig. 1.

For an equilibrium, $r_{e}$, to be asymptotically stable, we require from standard linearization techniques that $F^{\prime}\left(r_{\mathrm{e}}\right)=f\left(r_{\mathrm{e}}\right)<1$. Geometrically this means that stable equilibria occur where $F$ cuts the $45^{\circ}$ line from above. Now consider reverse bandwagon effects. Assume for each individual not

\footnotetext{
${ }^{1}$ Some loss of generality results from the restriction to one unit, but many commodities fit, e.g., those whose use is steady and limited within each time period (such as laundry detergent) and those that are a flow of services that one either has or does not (such as cable TV, a restaurant meal, a chauffeur). For many durables, high expense or rapidly diminishing marginal utility beyond the first unit help limit purchases to one per period, as with autos, dishwashers and solar collectors. We neglect the time distribution of product failure as a determinant of durable goods purchases, and for non-durables we neglect stockpiling.
} 


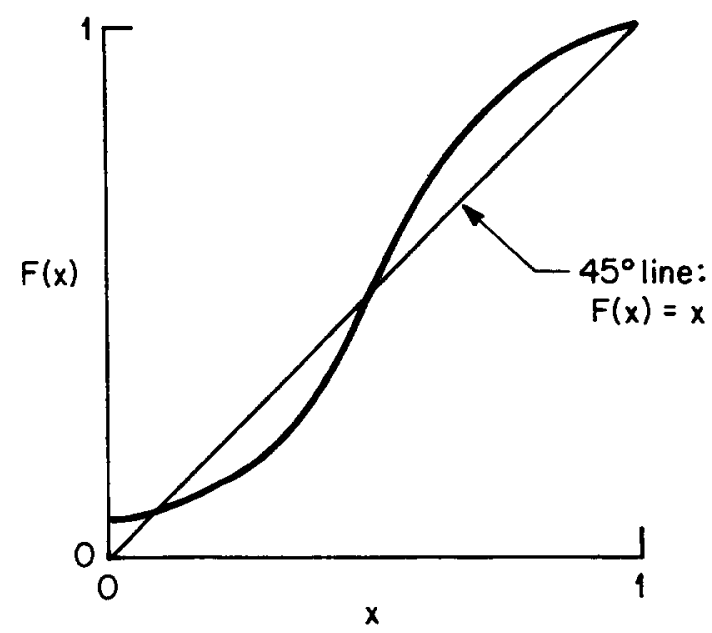

Fig. 1. $F(x)$ : Cumulative distribution function of thresholds.

only a threshold beyond which he will buy, but also a different kind of threshold, greater than the first, beyond which he will stop buying. One may need to see $20 \%$ of one's friends with push-button phones before succumbing to modern technology, but when $90 \%$ have them, the old rotary dial may develop perverse attractions.

The distributions of lower thresholds, $f_{\mathrm{L}}$, and of upper thresholds, $f_{\mathrm{U}}$, are the marginal densities of a joint density function of lower and upper thresholds, $f_{\mathrm{L}, \mathrm{U}}$, whose c.d.f. is $F_{\mathrm{L}, \mathrm{U}}$. This $f$ has the obvious restriction that for each individual the lower threshold must be less than the upper, so that the bivariate density is defined not over the unit square but only over its upper left triangle. Integrations of the marginal densities gives $F_{\mathrm{L}}$ and $F_{\mathrm{U}}$, the c.d.f.s of lower and upper thresholds, respectively. ${ }^{2}$

Suppose that at time $t, 45 \%$ of men buy narrow ties, and $60 \%$ have lower thresholds for such purchases of less than or equal to $45 \%$, and $25 \%$ have such upper thresholds. This $25 \%$ must be a subset of the $60 \%$, since one's lower threshold is less than one's upper; thus, at $(t+1)$ this subset will be deactivated, leaving only the balance, $35 \%$, to buy narrow ties. Buyers at

\footnotetext{
${ }^{2}$ For simplicity we depict situations where all individuals have a lower and upper threshold. But some individuals may not purchase regardless of how many others do so. We assign them a lower threshold of $100 \%$ : they buy only when everyone - including themselves - has bought, i.e., they will not buy. Since the behavior of such individuals is constant it does not enter the formal dynamic analysis. If there are others who will not stop buying no matter how many others buy, there is no upper threshold number to assign them and this shifts the formalities: $F_{\mathrm{U}}$ is no longer a distribution function since $F_{\mathrm{U}}(1)<1$, and then $G(1)>0$ rather than $=0$ as in fig. 2 . This shift does not affect our subsequent analysis. An alternate account that assigns numerical description to every individual associates with each a band of values, $\left[l_{i}, u_{i}\right]$ : one buys iff the fraction of buyers in the previous period is contained in the band. Those without upper thresholds have band $\left[l_{i}, 1\right]$, those with lower thresholds of $100 \%,[1,1]$. 'Contrarians', who buy iff no one else did, have band $[0,0]$; 'instigators', who buy first, but stop after some level of others' buying, have $\left[0, u_{i}\right]$; and the impervious, who buy under all conditions, regardless of what others do, have $[0,1]$.
} 
$(t+1)$ are just those whose lower but not yet their upper threshold has been exceeded - exactly the difference between the two c.d.f.'s. Thus

$$
r(t+1)=G(r(t))=F_{\mathrm{L}}(r(t))-F_{\mathrm{U}}(r(t))
$$

Note that with the substitution of $G$ for $F$, this is formally identical to eq. (1), so stability analysis is also the same, with asymptotic stability iff $G^{\prime}\left(r_{e}\right)$ lies between -1 and +1 . Fig. 2 indicates that $G$, being a difference between two c.d.f.'s, need not be monotonic. It may be instead, for example, single-peaked.

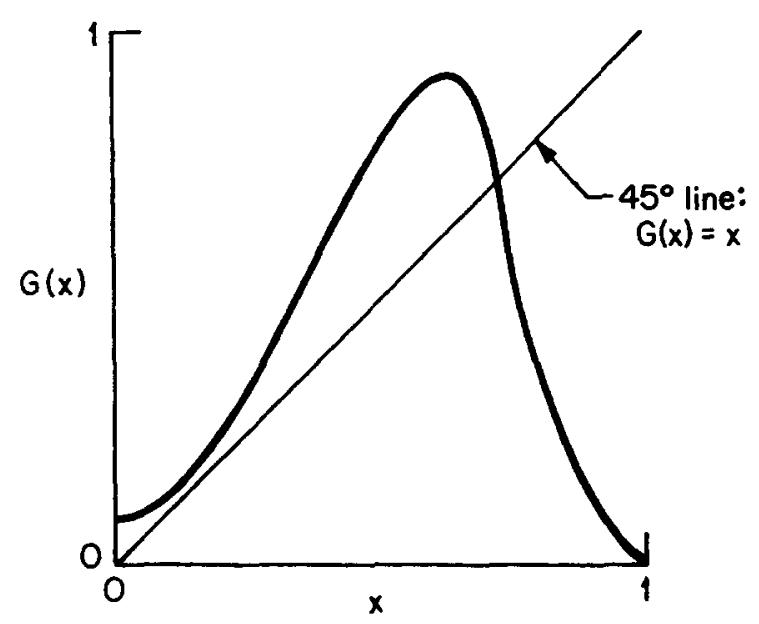

Fig. 2. $G(x)$ : The difference between two cumulative distribution functions, $F_{\mathrm{L}}(x)$, the c.d.f. of lower thresholds, and $F_{\mathrm{U}}(x)$, the c.d.f. of upper thresholds.

\section{Demand schedules for bandwagon effects}

To construct demand schedules requires some systematic relation between prices and thresholds. Thus far we have fixed prices, but thresholds may be expected to change when prices do. When personal computers were astronomically expensive, it is likely that people were influenced by the purchases of others, but with very high thresholds. At lower prices the previously insensitive were willing to buy when a much smaller number of others than before did so. For a given product, assume a different threshold distribution for each possible price, and, except for boundary constraints, a monotonic relation between price changes and those in thresholds - i.e., all individuals have lower thresholds at lower prices and the difference in thresholds is greater the greater that in price. A family of c.d.f.'s results, as in fig. 3, each keyed to a particular price, with prices declining from southeast to northwest, so $P_{0}>P_{1}$, etc. Monotonicity guarantees that each c.d.f. that represents thresholds for a lower price than another will lie strictly above it (except at unity where all c.d.f.'s must converge). As with indifference maps, then, between any pair of curves lie an infinite number of additional ones, each 


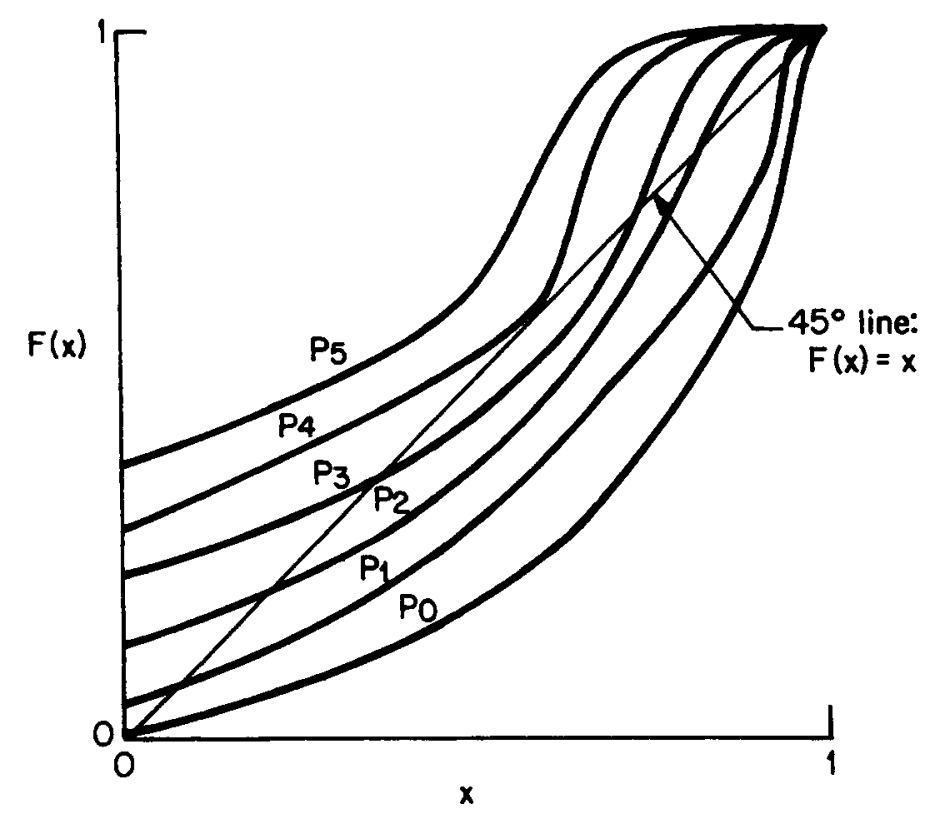

Fig. 3. Cumulative distribution functions of thresholds displayed for various product prices. Note that $P_{0}>P_{1}>P_{2}$, etc.

corresponding to some minute gradation in price; a curve is then a crosssection of the surface representing the function $F(x, p)$, where $F_{x}>0$ and $F_{p}<0$.

Assume for now a perfectly elastic supply of goods, so that any price changes will be exogeneous. For price $p$, intersections of the corresponding threshold distribution with the $45^{\circ}$ line are equilibrium quantities purchased at that price, and so are logical candidates for points on a demand schedule. But demand at some prices may then not be determinate, since there can be multiple equilibria, as between price $P_{0}$ and $P_{5}$ in fig. $3 .^{3}$ One way to assess which equilibrium number of purchases would be made at price $p$ is to suppose that the product is new so that initial purchases are zero. It is then plausible that the system will proceed to the smallest stable equilibrium. Since stable equilibria are those that cut the $45^{\circ}$ line from above, and the equilibrium number of purchasers has been equated by hypothesis to the quantity purchased, we may graph in fig. 4 a demand schedule from the surface, $F$, implied in fig. 3 . In this schedule, demand is everywhere determinate, but there is a sharp discontinuity. At price $P_{0}$ or higher, sales are zero because, at these prices, there are no zero threshold individuals who buy even when no others do so - and so zero purchases is the only stable equilibrium. Between prices $P_{0}$ and $P_{5}$ is a typical, downward-sloping demand curve. The amount sold increases gradually as prices decline,

\footnotetext{
${ }^{3}$ The curves in fig. 3 have the qualitative features of cubic c.d.f.s, corresponding to quadratic threshold probability densities: most thresholds are moderate, tailing off to smaller numbers of low and high values. Granovetter and Soong $(1983,1984)$ show that a wide class of parameterizations for such second-order polynomial densities leads to these multiple intersections.
} 


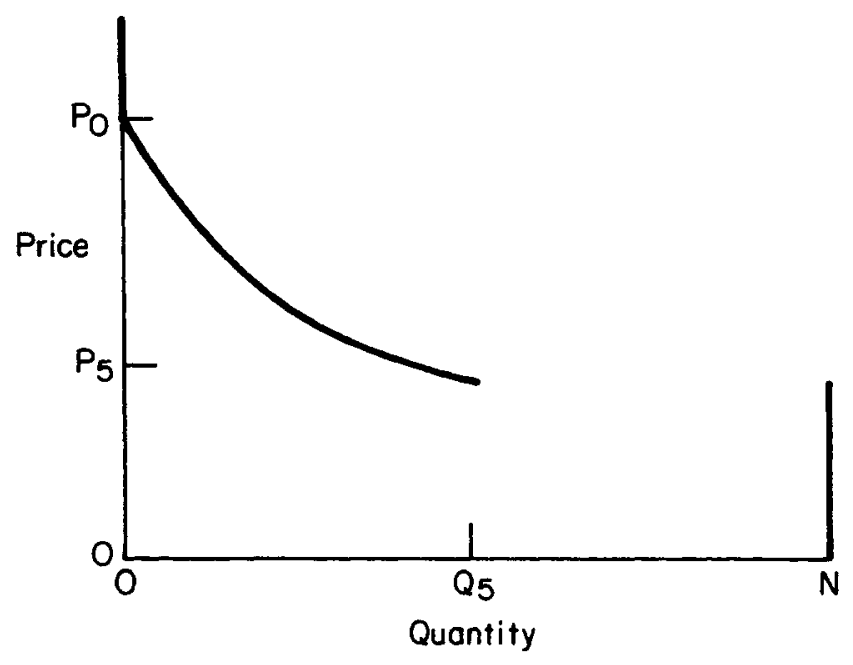

Fig. 4. A demand schedule corresponding to the threshold distributions of fig. 3, and the assumption that the minimum stable equilibrium of c.d.f.s in fig. 3 represents quantity purchased.

following the lower intersection of the c.d.f.'s up the $45^{\circ}$ line in fig. 3 , until price $P_{5}$, where the c.d.f. lies tangent to that line. Any further decline in price then results immediately in a large increase in sales to $N$, the population size, as the lower intersection of the c.d.f. with the $45^{\circ}$ line is lost and unity is the only stable equilibrium. ${ }^{4}$ Quantities between $Q_{5}$ and $N$ will not be sold at any price.

If we drop the condition of a new product (zero initial purchases) we must draw a more general demand curve with all stable and unstable equilibria. It is geometrically clear in fig. 3 that as prices decline, the location of stable equilibria (those cutting the $45^{\circ}$ line from above) will move higher; thus, except for boundary constraints, stable equilibria will yield standard, downward-sloping demand-schedule segments. Conversely, unstable equilibria, (those cutting the line from below) must produce upward-sloping segments. Since we would not expect to observe unstable equilibria in practice - they repel any nearby trajectory - this poses no contradiction to the usual patterns of demand. But graphing all points together, stable equilibria in solid lines and unstable in dotted, as in fig. 5, shows more complex discontinuities than in fig. 4. For prices between $P_{0}$ and $P_{5}$ we have the stable equilibrium shown in fig. 4 and also another at $N$, separated from the first set by unstable equilibria. At $P_{4}$, for example, the initial ('new product') equilibrium is $Q_{4}$. But if some perturbation, such as confusion about how many other buyers there were, pushed the quantity sold beyond the unstable equilibrium $Q_{U}$, the amount sold would move up to the second stable equilibrium of $N$, whose global stability is greater than that of $Q_{4}$. An industry aware of this might offer a brief price reduction calculated to push

\footnotetext{
${ }^{4}$ The tangency point occurs at a double root of the cubic, and curves beyond are those where two of the three solutions have shifted from real to imaginary.
} 


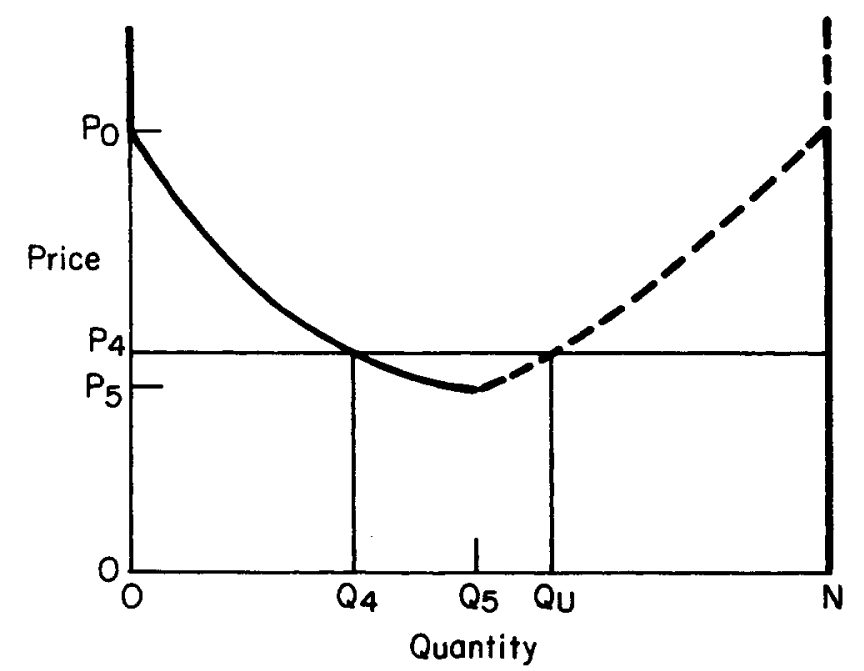

Fig. 5. A demand schedule corresponding to the the threshold distributions in fig. 3, showing stable equilibria in solid lines, unstable in dotted.

consumption beyond the unstable equilibrium, knowing that the price could then be restored to $P_{4}$ - or even pushed up almost to $P_{0}$ - without any loss of the new sales level, $N .^{5}$

Figs. 4 and 5 are drawn to correspond to the quadratic densities and cubic c.d.f.'s of fig. 3. If threshold distributions were such that only a single equilibrium obtained over the whole range of price changes, the resulting demand curve would be conventional; if the distributions were characterized instead by a series of hills and valleys, corresponding demand schedules would have more discontinuities and separable regions. Thus, a number of qualitatively different demand situations are consistent with bandwagon effects, depending on the exact details of interdependencies.

\section{Mixtures of bandwagon and reverse bandwagon effects}

We now treat mixtures of forward and reverse bandwagons, noting that the pure reverse bandwagon is a limiting case of a mixture, viz. where all bandwagon (lower) thresholds are 0 or $100 \%$ - prices may affect the initial buying decision but the behavior of others does not. Mixtures can be analyzed with the combination of lower and upper thresholds described by eq. (2).

Assume again that lower thresholds change in the same direction as prices; the opposite assumption seems apt for upper thresholds. Imagine that, at the prevailing price, you would end your subscription to cable TV when $90 \%$ of your friends had subscribed. If rates are cut, this 'snob' behavior will seem

\footnotetext{
${ }^{5}$ Note, however, that at prices near $P_{0}$, risks increase since the unstable middle equilibrium is closer than at $\boldsymbol{P}_{4}$ and even small perturbations may propel the system to the lower stable equilibrium that is near zero.
} 
less compelling; your upper threshold may move to $95 \%$. But if rates increase, the prospect of gaining status by abandoning the living room for plays and concerts may seem alluring when only $80 \%$ of others sit mesmerized by their home box office. ${ }^{6}$

To suppose that increases in price raise lower thresholds and depress upper ones, and that price decreases depress lower thresholds and raise upper ones has some face validity since all these changes make purchases move inversely to price changes. We again assume a monotonic relation between the size of price changes and those of thresholds.

These assumptions imply that an increase in price narrows the distance between upper and lower threshold distributions and a decrease widens it. In terms of c.d.f.'s, $\partial F_{\mathrm{L}} / \partial p<0$ and $\partial F_{\mathrm{U}} / \partial p>0$. Fig. 6a shows the upper and lower c.d.f.'s for two prices, $P_{0}$ and $P_{1}$, where $P_{0}$ is the larger, as before; the corresponding difference functions, $G_{0}$ and $G_{1}$ are displayed in fig. $6 \mathrm{~b}$. As with the map of fig. 3, for pure bandwagon effects, an infinitely dense map of such difference curves can be assumed, where each curve corresponds to a fixed commodity price. Declines in price both steepen the $G$ curve and increase the point of intersection with the $45^{\circ}$ line. This may at first appear to yield simply the standard downward-sloping demand schedule. But if $G$ intersects the line with slope of absolute value greater than unity, the indicated equilibrium is unstable, repelling all nearby trajectories, and would thus not be observed.

This situation did not arise in pure bandwagon effects since $F_{\mathrm{L}}$ is a distribution function, monotonically increasing; any crossing of the $45^{\circ}$ line

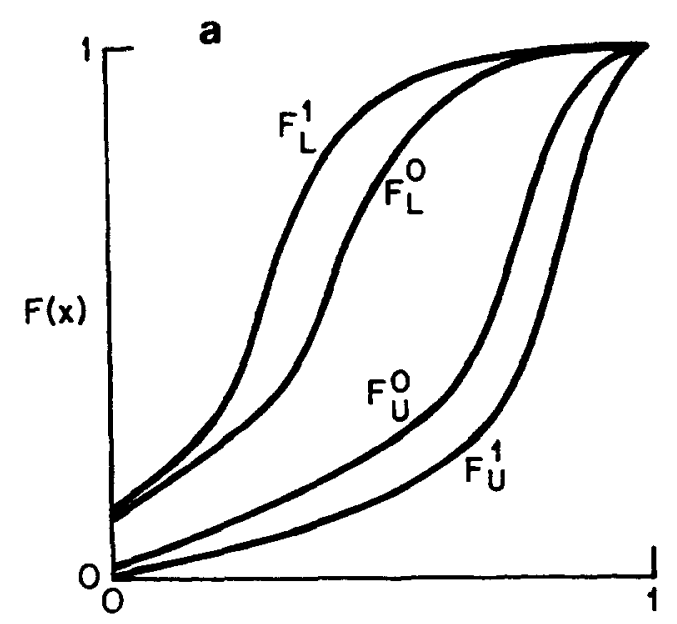

Fig. 6a. Cumulative distribution functions for lower and upper thresholds, shown for two prices, represented by superscripts 0 and 1 . Note that price 0 is greater than price 1 .

\footnotetext{
${ }^{6} \mathrm{We}$ assume the absence of 'money illusion' - valuation of goods in part because of their cost, independent of utility conferred. In particular, if 'Veblen effects' [Leibenstein (1976, pp. 62-63)] are present, where people want expensive commodities for 'conspicuous consumption', then the effects of a price increase on upper thresholds are ambiguous since it makes the product more attractive.
} 


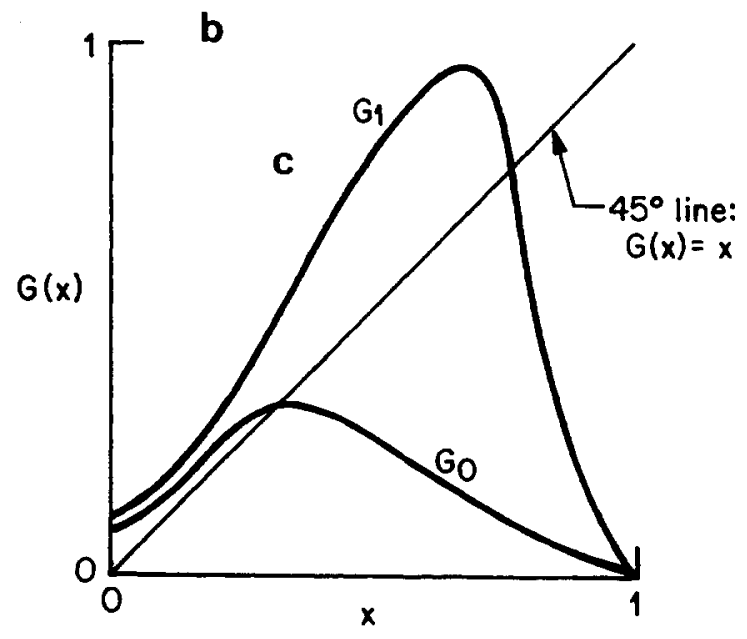

Fig. $6 \mathrm{~b}$. Two difference functions, $G(x)$, representing the differences between lower and upper c.d.f.s shown in fig. $6 \mathrm{a}$. $G_{0}$ is the difference between $F_{\mathrm{L}}^{0}$ and $F_{\mathrm{U}}^{0}$, and similarly for $G_{1}$.

from above has slope less than unity and is then stable, with at least one such crossing guaranteed. But the functions $G$ are differences of distribution functions, and no such guarantees obtain. Where the sole intersection of $G$ with the line has slope greater than unity, as for $G_{1}$ in fig. $6 \mathrm{~b}$, there is no stable equilibrium, and the system enters an oscillatory mode. Where the slope first exceeds unity, the single equilibrium bifurcates into an oscillation of period two about the now unstable equilibrium, and the system cycles back and forth between these two values. This oscillation is asymptotically stable: if pushed away from either value, the system returns to this oscillation. As the slope steepens, the outcome bifurcates from stable oscillation among two values to that among four, then eight, sixteen, etc., until a 'critical' steepness is reached, beyond which lies what has been called the 'chaotic' region. There, depending on the details of $G$, one finds either stable cycles of any period (not necessarily exponents of 2) or completely aperiodic ('chaotic') behavior of the system [see May (1976), May and Oster (1976)].

Within the chaotic region, May indicates that for many 'smooth and "sensible" functions... for any specified parameter value there is one unique cycle that is stable and that attracts essentially all initial points' [May (1976, p. 462)]. That is, the measure of chaotic trajectories can be zero. But this is not necessarily the case for all relevant functions: Day and Shafer (1985), for example, use a piecewise function in Keynesian dynamics to show that the chaotic set may, under fairly simple assumptions, not only have nonzero measure but even dominate outcomes. [For other illustrations of chaotic dynamics in economic contexts see Day (1982 and 1983) and Benhabib and Day (1981)]. But even if chaotic trajectories did have measure zero, most cycles in that region would have a large period, 'and moreover it will typically take many thousands of generations before the transients associated 
with the initial conditions are damped out' [May (1976, p. 464)]. It is then 'essentially irrelevant whether the solution is aperiodic or periodic with long period; these are likely to be indistinguishable in practice, and small fluctuations in [steepness of slope] or in data collected are likely to blur the distinction, even if it did exist.' [May and Oster (1976, p. 595)].

In fig. 7a we construct a demand schedule by plotting against each price all equilibrium amounts of the good purchased at that price, assuming again a perfectly elastic supply of goods. For prices higher than $P_{1}$, where the $G$ curve first crosses with slope steeper than minus one, the curve has the standard shape. As the price declines below $P_{1}$, the stable solution $Q_{s}$ bifurcates into two solutions, and the system oscillates back and forth between those. (The standard demand schedule diagram indicates such dynamics poorly; fig. $7 \mathrm{~b}$ shows, for various prices corresponding to fig. $7 \mathrm{a}$, exact time trajectories of quantity demanded at equilibrium.) At points near $P_{1}$, the oscillation is hardly noticeable as both points are quite near $Q_{1}$, but as price declines further the two move further apart until there is still another bifurcation. In such systems, the parameter windows - here, price reductions - required to yield successive bifurcations become increasingly smaller until a critical point, $P^{*}$, is reached, beyond which bifurcations halt and the chaotic region described above is entered [May (1976)]. Thus, quantities sold at these prices would fluctuate in unpredictable ways even though the underlying assumptions are completely deterministic. ${ }^{7}$

The curves in figs. 6 and 7 depict unimodal difference functions, $G$, that cross the $45^{\circ}$ line with sufficiently steep slopes to throw the system into the chaotic region. If underlying thresholds were such that slopes never exceeded unity, resulting demand curves would be conventional. If the Gs were multimodal, analysis would be correspondingly more complex, given the possibility of multiple intersections of $G$ with the $45^{\circ}$ line. As with pure (forward) bandwagon effects, a variety of situations is possible depending on the exact details of interdependencies.

\section{Models with supply response}

For long-run dynamics it is not too troublesome to assume a perfectly elastic supply of goods; but the responses of consumers to the purchases of others, that generate equilibrium levels of demand in this model, occur in the short run. Specifically, we have argued that when $r(t)$ individuals buy at time $t, F(r(t))$ will do so at the same price at time $(t+1)$. For this price to remain

\footnotetext{
${ }^{7}$ Note that at each bifurcation point, solutions are shifted from solid to dotted lines, since at all lower prices these continue to be equilibrium solutions or cycles, but are unstable. Bifurcations beyond $2^{4}$ solutions are not shown on the diagram, as the increasing narrowness of parameter windows, which approach zero in size as price approaches $P^{*}$, cannot readily be represented.
} 


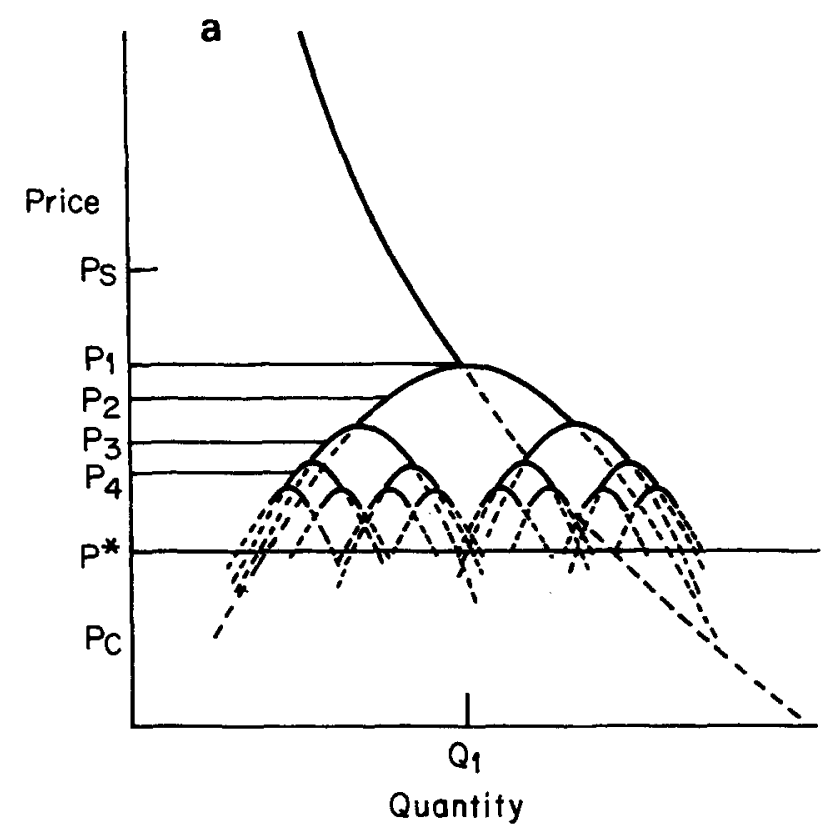

Fig. 7a. A demand schedule corresponding to the family of $G$ curves implicit in fig. $6 \mathrm{~b}$, where both bandwagon and reverse bandwagon effects are present. Stable equilibria and cycles are shown in solid lines, unstable in dotted.

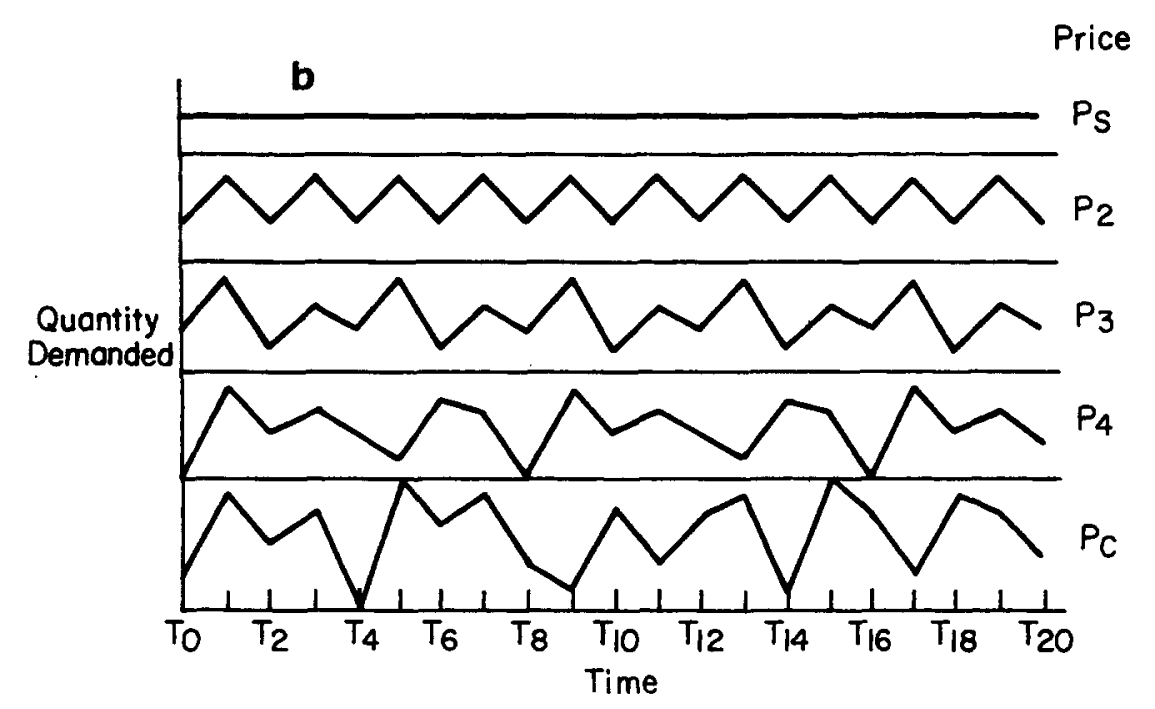

Fig. $7 \mathrm{~b}$. The time path of consumer demand at equilibrium, for various prices indicated in fig. 7a.

unchanged does require perfectly elastic supply since if $F(r(t))$ units were more or less than what was available at the prevailing price, excess supply or demand would exert pressure on that price. But a price change, by hypothesis, would throw consumers onto a different threshold distribution, requiring more complex dynamic assumptions. 
Our treatment of supply is schematic; we merely allow any non-horizontal supply schedule. ${ }^{8}$ Begin with forward bandwagons, and assume some supply function $S(p(t))$. Since the $r(t+1)=F(r(t))$ units that consumers want to buy at $(t+1)$ may be more than what is supplied at $p(t)$ it represents not actual purchases, as above, but rather demand. We thus relabel the parameter $r(t)$ as $D(t)$. If $D(t)>S(p(t))$ we have excess demand and can expect a higher price in the next time period, shifting consumers to a lower threshold curve in the family depicted by fig. 3 , and conversely for $D<S$. This requires a new parameter and equation in the model, to represent price movements

$$
p(t+1)=p(t)+H[D(t)-S(p(t))]
$$

The argument of $H$ is excess demand or supply at time $t$, and we assume $H(0)=0$. Note that this equation is a discrete-time analogue of the priceadjustment equation suggested by Samuelson (1947, p. 263).

The assumptions about demand are unchanged from the model with perfectly elastic supply, but we rewrite eq. (1) with $r$ relabeled as $D$ and with its dependence on price made explicit

$$
D(t+1)=F[p(t+1), D(t)]=F\{p(t)+H[D(t)-S(p(t))], D(t)\} .
$$

That is, price in the next period is determined by current price and current excess supply or demand; demand in the next period is determined by consumers' responses to current demand and by price in the next period. Since the next period's price is entirely determined by current price, demand and supply, eq. (3) can be substituted into eq. (4) as shown, and all arguments of the r.h.s. of (4) are in $t$, yielding a system of two coupled, firstorder difference equations.

The account seems intuitively plausible. In deciding on current price, suppliers have little to go on but the level of excess demand or supply in the previous period. Consumers are sensitive to current price but cannot see current demand any better than suppliers can since they are in the process of producing it by their separate decisions; they thus respond to the previous level of demand, each according to his threshold.

Each equation generates one equilibrium condition. The first is that supply equal demand, the second that $D(t+1)=D(t)=F(D(t))$. Note that the demand curve already drawn in fig. 5 is exactly the locus of all points satisfying the second condition. When a supply curve is drawn in, then, any intersection of that curve with the demand curve will satisfy both conditions; the set of such intersections must then be the set of all equilibrium points for the

\footnotetext{
${ }^{8} \mathrm{~A}$ fully symmetric treatment would allow bandwagons for supply as well as for demand. In the game-theoretic literature on oligopoly and in some sociological treatments [White (1981)], suppliers' decisions are keyed to one another's. But we abstract away from such effects here.
} 
two-equation system. In this respect, the introduction of inelastic supply has not altered the earlier analysis.

But the stability of equilibria need not carry over to the extended model. We proceed by linearizing the system of eqs. (3) and (4). The matrix of first partial derivatives, evaluated at some equilibrium $(p, D)$, where $F_{p}$ and $F_{D}$ are the relevant partial derivatives of $F$, evaluated at $(p, D)$, is

$$
\left[\begin{array}{cc}
1-H^{\prime}(0) S^{\prime}(p) & H^{\prime}(0) \\
F_{p}-F_{p} H^{\prime}(0) S^{\prime}(p) & F_{p} H^{\prime}(0)+F_{D}
\end{array}\right]
$$

For asymptotic stability both eigenvalues must have absolute value less than unity. Setting the determinant of the matrix to zero yields the following characteristic equation:

$$
0=L^{2}+L\left[-1+H^{\prime}(0) S^{\prime}(p)-F_{p} H^{\prime}(0)-F_{D}\right]+\left[F_{D}-F_{D} H^{\prime}(0) S^{\prime}(p)\right]
$$

Note that this is in the form $0=a L^{2}+b L+c$ and that $a=1$. There is no obvious way to factor (5), and rather than derive cumbersome general expressions from the quadratic formula, we note that both eigenvalues are less than unity iff the following three conditions, expressed in terms of the quadratic coefficients, are met: ${ }^{9}$

$$
\text { (i) }-1<c<+1, \quad \text { (ii) }-1<b+c, \quad \text { (iii) }-1<c-b \text {. }
$$

Graphing these conditions together in fig. 8, we see that the region of stability is given by the cross-hatched triangle.

Condition (ii) is equivalent to $H^{\prime}(0)\left[S^{\prime}(p)\left(1-F_{D}\right)-F_{p}\right]>0$. Since the forward bandwagon is monotonically increasing, $F_{D}$ is always positive; $F_{P}$ is negative since an increase in price moves consumers to a lower cumulative threshold distribution. Then if $H^{\prime}(0)$ is positive - reasonable for many priceadjustment functions ${ }^{10}$ - and $S^{\prime}(p)$ is positive - the supply curve slopes up at

${ }^{9}$ These conditions are derived as follows: (i) From the characteristic eq. (5) we seek solutions $L_{1}$ and $L_{2}$ such that $0=L^{2}+b L+c=\left(L-L_{1}\right)\left(L-L_{2}\right)$. Multiplying out yields $L^{2}+\left(-L_{1}-L_{2}\right) L+$ $L_{1} L_{2}$. Thus the product of the roots equals the coefficient $c$, and it is necessary but not sufficient for stability that $c$ have absolute value less than unity; otherwise one eigenvalue must be greater than or equal to unity. (ii) We require further that the larger root be less than +1 and the smaller greater than -1 . The first of these conditions is that $\left[-b+\left(b^{2}-4 c\right)^{1 / 2}\right] / 2<1$, so $\left(b^{2}-4 c\right)^{1 / 2}<2+b$. Squaring both sides, the condition is equivalent to $-1<b+c$. (iii) For the smaller eigenvalue we require that $-1<\left[-b-\left(b^{2}-4 c\right)^{1 / 2}\right] / 2$, that is, $b-2<-\left(b^{2}-4 c\right)^{1 / 2}$. Squaring this inequality yields $-1<c-b$. The two eigenvalues may be either both real or both complex. If the latter, they occur in conjugate pairs and thus both have the same absolute value; then any pair that meets one of conditions (ii) and (iii) must also meet the other.

${ }^{10} \mathrm{It}$ is also plausible for a price-adjustment function that $H^{\prime}(0)=0$, as where the size of the adjustment declines more rapidly as excess supply or demand becomes smaller. An example 




Fig. 8. Cross-hatched triangle represents values of the quadratic coefficients, $b$ and $c$, such that both eigenvalues have absolute value less than unity, and the system is stable. Note that $b=$ $\left.\left(-1+H^{\prime}(0) S^{\prime}(P)-F_{p} H^{\prime}(0)-F_{D}\right)\right)$ and $c=F_{D}-F_{D} H^{\prime}(0) S^{\prime}(p)$.

the equilibrium price - then this condition is met if $F_{D}<1$. That is, this stability condition is always met when the threshold curve corresponding to price $p$ crosses the $45^{\circ}$ line from above, at the equilibrium value $D$. Note that the locus of all such crossings is just that given in fig. 5 by solid lines.

To sum up: the demand curve is unchanged when inelastic demand is introduced, being the locus of all points that depict demand for a given equilibrium price. There is a parameter region within which the pricequantity equilibria resulting from the system of eqs. (3) and (4) are asymptotically stable. As with perfectly elastic supply, we may have multiple equilibria, as can be seen by drawing upward-sloping supply curves through the demand curve of fig. 5. Because stability now depends on the value of four separate derivatives $\left(F_{d}, F_{p}, S^{\prime}\right.$ and $\left.H^{\prime}\right)$, we cannot determine it from inspection of the supply-demand cross. Some of the equilibria that were unstable under perfectly elastic supply (whose locus is given by dotted lines in fig. 5) may be stable in the extended system; conversely, some stable equilibria (solid lines in fig. 5) may now be unstable. These latter cross the $45^{\circ}$ line with slope less than unity; but this now guarantees only that one eigenvalue is less than +1 . The second could still be less than -1 , destabilizing the system. Moreover, systems of nonlinear equations are much more prone to fall into cycles, or regions with chaotic dynamics, than are

would be $H(x)=x^{3}$. But if $H^{\prime}(0)=0$, the two eigenvalues are $F_{\mathrm{D}}$ and 1 . The latter eigenvalue is a boundary case and renders the linearized stability analysis uniformative; more detailed analysis of the particular equations would then be required to determine the stability properties of an equilibrium. 
single such equations [Kaplan and Yorke (1979), Marotto (1978)], and some parameterizations might yield this confusing dynamic behavior even in the absence of upper thresholds. This possibility was excluded for forward bandwagons with perfectly elastic supply because the single difference equation (1) was monotonically increasing, with no possibility of the 'tunable hump' required for chaotic dynamics [May (1976)]. Finally, even the move from one to two dimenions alters the conclusion that chaotic trajectories have zero measure for many analytical functions, and reduces the level of nonlinearity required for such phenomena to occur [May $(1976$, p. 466)]. Fig. 5 must then be only a partial picture of system outcomes, since there may also be stable cycles and aperiodic behavior.

Mixtures of forward and reverse bandwagons can also be analysed with eqs. (3) and (4), everywhere substituting $G$ for $F$, where $G$ remains the difference between lower and upper cumulative distributions. Since any equilibrium must then satisfy $D(t+1)=D(t)=G(D(t))$, the downward sloping line of fig. 7a's demand curve continues to be the locus of all possible equilibria that occur at a point in the state space, rather than in a cycle. But the solid and dotted lines no longer indicate which equilibria are stable, and the situation is less clear than for pure forward bandwagons since $G_{D}$ may be positive or negative, unlike $F_{D}$ which - being the slope of a c.d.f. - must be positive. The system may then be in stable equilibrium, oscillate in stable cycles or enter aperiodic, 'chaotic' trajectories. If cycles occur they have no necessary relation to those shown in figs. $7 \mathrm{a}$ and $7 \mathrm{~b}$, since the latter are cycles of demand only; in the two-equation system, cycles involve changes in each period of both demand and price.

\section{Summary}

The models in this paper illustrate the implications of some simple assumptions about interpersonal effects on consumption where buying depends in part on how many others have previously bought. Rather than examine utility functions directly, we make assumptions about individual differences in sensitivity to others' behavior and about the effect of price changes on these sensitivities, or 'thresholds'. The resulting demand schedules and their interactions with supply show that even in the presence of perfect information, profit-maximizing firms and utility-maximizing individuals, unstable, oscillatory and even completely indeterminate market situations may result. These outcomes result entirely from the interpersonal effects.

We make no attempt here to discuss the peculiar features of industrial organization that might evolve in attempts to reduce such uncertainties. To do so in any detail would be a larger and different project, though one that the present models should illuminate. 


\section{References}

Benhabib, Jess and Richard Day, 1981, Rational choice and erratic behavior, Review of Economic Studies 48, 459-471.

Day, Richard, 1982, Irregular growth cycles, American Economic Review 72, 406-414.

Day, Richard, 1983, The emergence of chaos from classical economic growth, Quarterly Journal of Economics 97, 201-213.

Day, Richard and Wayne Shafer, 1985, Keynesian chaos, Journal of Macroeconomics, forthcoming.

Duesenberry, James, 1949, Income, savings and the theory of consumer behavior (Harvard University Press, Cambridge, MA).

Granovetter, Mark, 1978, Threshold models of collective behavior, American Journal of Sociology 83, 1420-1443.

Granovetter, Mark and Roland Soong, 1983, Threshold models of diffusion and collective behavior, Journal of Mathematical Sociology 9, 165-179.

Granovetter, Mark and Roland Soong, 1984, Threshold models of collective behavior, Draft monograph, ch. 1 (State University of New York at Stony Brook, NY).

Kaplan, James and James Yorke, 1979, Chaotic behavior of multidimensional difference equations, in: $H$. Peitgen and $H$. Walther, eds., Functional differential equations and approximations of fixed points, Lecture Notes in Mathematics 730 (Springer, Berlin).

Leibenstein, Harvey, 1950, Bandwagon, snob and Veblen effects in the theory of consumer demand, Quarterly Journal of Economics 64, 183-207.

Leibenstein, Harvey, 1976, Beyond economic man: A new foundation for microeconomics (Harvard University Press, Cambridge, MA).

Marotto, Frederick, 1978, Snap-back repellers imply chaos in $R^{n}$, Journal of Mathematical Analysis and Applications 63, 199-223.

May, Robert, 1976, Simple mathematical models with very complicated dynamics, Nature June $10,261,459-467$.

May, Robert and George Oster, 1976, Bifurcations and dynamic complexity in simple ecological models, American Naturalist 110, 573-599.

Nelson, Philip, 1970, Information and consumer behavior, Journal of Political Economy 78, 311-329.

Samuelson, Paul, 1947, Foundations of economic analysis (Harvard University Press, Cambridge, MA).

Veblen, Thorstein, 1899 [1934], The theory of the leisure class (Modern Library, Random House, New York).

White, Harrison, 1981, Where do markets come from? American Journal of Sociology 87, 517547. 